\title{
Fatal adverse events with molecular targeted agents in the treatment of advanced hepatocellular carcinoma: a meta-analysis of randomized controlled trials
}

This article was published in the following Dove Press journal:

Drug Design, Development and Therapy

\author{
Xiaofei $\mathrm{Li}^{\prime}$ \\ Jia Wan ${ }^{2}$ \\ Zhenping $\mathrm{Wu}^{3}$ \\ Juncai Tu' \\ Yongtao $\mathrm{Hu}^{\prime}$ \\ Shuang $\mathrm{Wu}^{\prime}$ \\ Lianqing Lou' \\ 'Department of Infectious Diseases, \\ Yiwu Central Hospital, Yiwu, China; \\ ${ }^{2}$ The First Hospital of Nanchang, \\ Nanchang, China; ${ }^{3}$ Gastroenterology \\ Department, Jinhua Central Hospital, \\ Jinhua, China
}

\begin{abstract}
Aims: Concerns have increased about the risk of fatal adverse events (FAEs) associated with molecular targeted agents (MTAs) in the treatment of advanced hepatocellular carcinoma (HCC). The purpose of this study is to investigate the overall incidence and risk of FAEs in advanced HCC with administration of MTAs by using a meta-analysis of available clinical trials.

Materials and methods: Electronic databases were searched for relevant articles before March 2017. Eligible studies were selected according to the Preferred Reporting Items for Systematic Reviews and Meta-Analysis (PRISMA) statement. Pooled incidence, Peto ORs and 95\% CIs were calculated according to the heterogeneity of selected studies.

Results: A total of 4,716 HCC participants from 10 randomized controlled trials (RCTs) were finally considered for this meta-analysis. The pooled incidence of death due to MTAs was $2.1 \%$ (95\% CI $1.6 \%-2.8 \%$ ) with a Peto OR of 1.79 (95\% CI $1.07-3.01 ; p=0.027)$ in comparison with controlled groups. Subgroup analysis according to biological agents showed that brivanib treatment in HCC patients significantly increased the risk of developing FAEs (Peto OR 3.97; 95\% CI 1.17-13.51; $p=0.028$ ) but not for sorafenib (Peto OR 1.78; 95\% CI 0.54-5.89; $p=0.34$ ) and other MTAs (Peto OR 1.43; 95\% CI 0.75-2.76; $p=0.28$ ). Sensitive analysis showed that the pooled results were influenced by removing each single trial. The most common causes of FAEs were hepatic failure (22.2\%) and hemorrhage (13.3\%), respectively.

Conclusion: Clinicians should be aware of the risks of FAEs during the administration of MTAs in advanced HCC patients, especially for patients with abnormal liver function. However, the use of sorafenib remains justified in its approved indications due to their potential survival benefits and limited toxicities.
\end{abstract}

Keywords: liver cancer, clinical trials, novel molecular agents

\section{Introduction}

Hepatocellular carcinoma (HCC) is one of the leading causes of cancer-related mortality in the world, with more than 696,000 deaths being identified annually. ${ }^{1,2}$ Globally, substantial geographic variation exists in incidence, with the highest incidence rates occurring in East and South-East Asia and in Middle and Western Africa. The management of HCC is a major health problem worldwide. Despite the implementation of screening programs for high-risk individuals, approximately $70 \%$ of HCC patients have intermediate-stage or advanced-stage disease at the time of diagnosis. The prognosis
Correspondence: Lianqing Lou

Department of Infectious Diseases, Yiwu Central Hospital, No 519 Nanmen Road,

Yiwu, Zhejiang 322000, China

Tel +8657985209617

Fax +86 57985209617

Email loulanqing2017@tom.com 
of advanced HCC patients is very poor, with a median overall survival (OS) time of about 7 months. ${ }^{3}$

In recent years, improved knowledge of hepatocarcinogenesis has led to the identification of novel drugs for the treatment of HCC. ${ }^{4-6}$ In general, these drugs target key molecules or signaling pathways which regulate cell growth and proliferation, angiogenesis, or invasion. ${ }^{7}$ Until now, sorafenib, an oral multi-targeted tyrosine kinase inhibitor (TKI), remains the only approved systemic treatment for unresectable advanced or metastatic HCC, due to its significant survival benefit in two large Phase III trials (SHARP and ORIENTAL). ${ }^{8,9}$ In addition to sorafenib, other TKIs, such as brivanib, sunitinib, or regorafenib, and an oral smallmolecular serine-threonine kinase inhibitor everolimus, have been in development to treatment HCC both in the first-line setting and for use following sorafenib failure. However, these signaling pathways play an important role in vascular function and physiological angiogenesis, thus inhibition of these pathways by these molecular targeted agents (MTAs) has been found to cause serious adverse events (AEs), including hepatic failure, bleeding, and thromboembolic events. ${ }^{10-12}$ In addition, several published meta-analyses have found that the use of these MTAs significantly increases the risk of developing fatal adverse events (FAEs) in cancer patients..$^{10,13-17}$ However, to our best knowledge, the overall incidence and risk of FAEs associated with MTAs in HCC patients remains undetermined. As a result, we conduct this meta-analysis of randomized controlled trials (RCTs) to investigate the overall incidence and risk of FAEs associated with MTAs in advanced HCC.

\section{Materials and methods}

\section{Data source}

Following the Preferred Reporting Items for Systematic Reviews and Meta-Analysis (PRISMA) statement, we performed an independent review of related citations from PubMed, Embase, and Cochrane Library electronic databases up to March 2017. ${ }^{18}$ The search keywords were "molecular targeted agents", "sorafenib", "sunitinib", "regorafenib", "ramucirumab", "brivanib", "everolimus", "pazopanib", "vandetanib", "fatal adverse events", and "randomized controlled trials". The search was restricted to clinical trials published in English. Additionally, relevant articles in the reference lists of recent meta-analyses that investigated MTAs in HCC patients were also searched. In order to avoid duplication, only the most complete, recent studies were considered for analysis.

\section{Clinical end points definition}

FAEs have been defined by the National Cancer Institute's Common Terminology Criteria for Adverse Events (CTCAE) as deaths occurring during a clinical trial as a result of exposure to an experimental drug. Most of the included studies did report the specifics of study deaths. For deaths unrelated to AEs, the guidelines required that any death occurring within 30 days of intervention be reported. We excluded events that were reported as related to disease progression, but included all events with unspecified attribution and included events regardless of attribution to treatment, provided that they were not related to disease progression. In rare situations, "Death NOS (not specified)" or "Sudden Death" was available when death could not be attributed to a CTCAE term.

\section{Study selection}

The primary objective of the present study was to evaluate the association between MTA treatment and treated related deaths in advanced HCC patients; therefore, only RCTs with a direct comparison between MTAs, alone or in combination with another anticancer agent and a controlled therapy were included. Articles were first screened on the basis of title and abstract to identify eligible RCTs. Phase I, single-arm Phase II trials and randomized trials with MTAs in both arms were excluded for analysis. RCTs reported as meeting abstracts without detailed data on AEs were also excluded. Clinical trials that met the following criteria were included: prospective RCTs that randomly assigned patients to MTAs treatment or controls; and with available data regarding incidence and cause of FAEs.

\section{Data extraction}

Data abstraction was conducted independently by two investigators, and any discrepancy between the reviewers was resolved by consensus. The numbers of FAEs and reported specific causes in both treatment and control arms were extracted from text or appendix of the trial publications. For multiple reports of the same trial, we used data from the longest follow-up. For all eligible trials, we also extracted the following information: first author's name, year of publication, trial phase, number of enrolled subjects, treatment arms, number of patients in treatment and controlled groups, median age, and median progression-free survival.

\section{Statistical method}

The primary summary measures were incidence, Peto ORs, and corresponding 95\% CIs. All statistical analyses 
were performed by using Version 2 of the Comprehensive MetaAnalysis program (Biostat, Englewood, NJ, USA). For the calculation of incidence, the number of patients with FAEs in the MTA group and the total number of patients receiving MTAs were extracted; the proportion of patients with FAEs and 95\% CI were derived for each study. To calculate Peto ORs, patients assigned to MTAs were compared only with those assigned to control treatment in the same trial. We used the Peto method to calculate ORs and 95\% CIs because this method provided the best confidence interval coverage when dealing with low event rates. ${ }^{19}$ Between-study heterogeneity was estimated using the $\chi^{2}$-based $Q$ statistic. ${ }^{20}$ The $I^{2}$ statistic was also calculated to evaluate the extent of variability attributable to statistical heterogeneity between trials. A statistical test with a $p$-value less than 0.05 was considered significant. Study quality was assessed by using the Jadad scale based on the reporting of the studies' methods and results. ${ }^{21}$

\section{Results}

\section{Search results}

We initially found 1,500 reports. After excluding review articles, Phase I studies, case reports, editorial, letters, commentaries, meta-analyses and systematic review (Figure 1), we selected 13 prospective clinical trials. Three RCTs were removed from the meta-analysis because both groups received MTAs treatment. ${ }^{22-24}$ Finally, a total of 10 RCTs were included for analysis in the present study. ${ }^{8,25-33}$ There

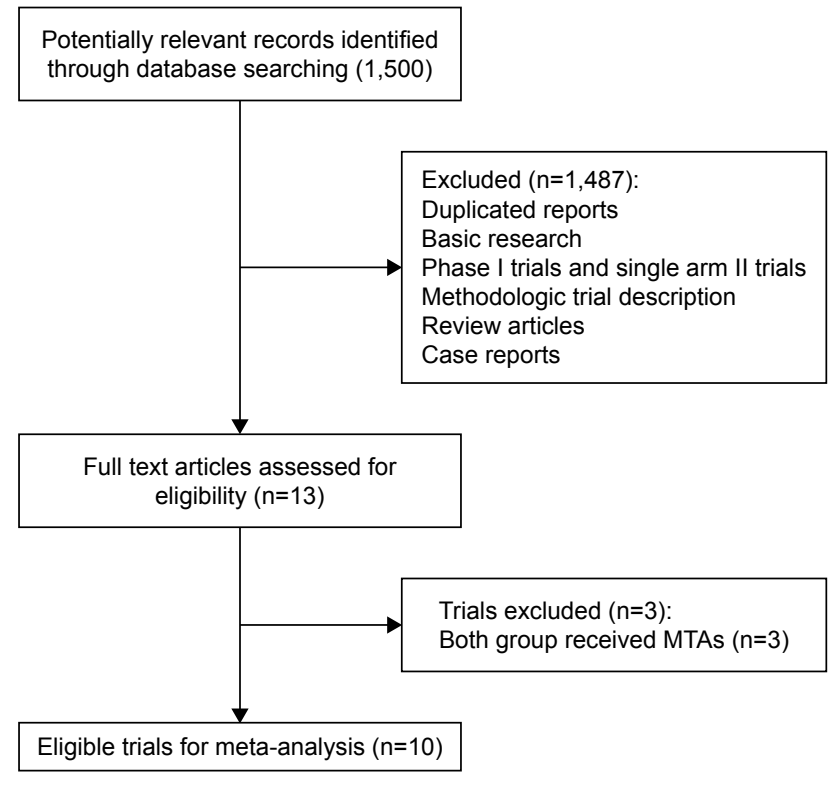

Figure I Studies eligible for inclusion in the meta-analysis. Abbreviation: MTAs, molecular targeted agents. were no FAEs observed in two included trials, conducted by Cheng et $\mathrm{al}^{8}$ and Kudo et al. ${ }^{26}$

\section{Study characteristics}

Ten trials including a total of 4,716 patients were included in the final analysis. Table 1 lists the baseline characteristics of patients and studies. The quality of each study was roughly assessed according to the Jadad scale. Ten trials were double blinded and placebo controlled, thus had a Jadad score of 5. Eight trials used MTAs as monotherapy, and two as add-on or combination therapy. MTA was compared with active controls or placebo/BSC alone in two and eight trials, respectively. Eight trials were Phase III randomized trials and the remaining two trials were Phase II clinical trials. FAEs were defined by the CTCAE version 3.0 or 4.0 criteria in all the included trials.

\section{Incidence and risk of FAEs with MTAs}

For calculating the overall incidence of FAEs associated with MTAs, a total of 2,652 HCC patients receiving MTAs were included. No FAEs were observed in two trials. The pooled incidence of FAEs related with MTAs was 2.1\% (95\% CI $1.6 \%-2.8 \%)$. For calculating the risk of FAEs associated with MTAs, a total of 4,716 patients were finally included in the present study. The pooled risk of developing FAEs was 1.79 (95\% CI 1.07-3.01, $p=0.027)$ in HCC patients receiving MTAs in comparison with controls (Figure 2). We also did a sensitivity analysis to examine the stability and reliability of pooled ORs by sequential omission of individual studies (the pooled results are shown after removing each indicated study in Figure 3). The leaveone-out analysis was performed by omitting one study in turn, as indicated in Figure 3. The results indicated that the estimate of the pooled ORs became non-significant after omitting the trials conducted by Llovet et $\mathrm{al}^{28}$ or Kudo et $\mathrm{al}^{27}$ (Figure 3). Both of these trials assessed the efficacy and toxicities of brivanib in the treatment of advanced HCC, which suggested that brivanib had more toxicities than the placebo. Then, we performed subgroup analysis according to the specific targeted drugs, and found that all of the subgroup analyses indicated a trend toward an increased risk of FAEs associated with MTAs in HCC patients. In addition, the use of brivanib in HCC significantly increased the risk of developing FAEs (Peto OR 3.97, $p=0.028$ ). In agreement with our result, higher incidences of treatment discontinuation from AEs was observed with brivanib versus sorafenib (43\% vs 33\%) in a large Phase III head-to-head trial for advanced HCC patients, although both brivanib 
Table I Baseline characteristic of the 10 trials

\begin{tabular}{|c|c|c|c|c|c|c|c|c|c|}
\hline Reference & Phase & Total & Treatment arms & $\begin{array}{l}\text { Median } \\
\text { age (years) }\end{array}$ & $\begin{array}{l}\text { Median } \\
\text { PFS }\end{array}$ & $\begin{array}{l}\text { Median } \\
\text { OS }\end{array}$ & $\begin{array}{l}\text { No for } \\
\text { analysis }\end{array}$ & FAEs & $\begin{array}{l}\text { Jadad } \\
\text { score }\end{array}$ \\
\hline \multirow[t]{2}{*}{ Cheng et $\mathrm{al}^{8}$} & III & 271 & Sorafenib $400 \mathrm{mg}$ bid po & 51 & 2.8 & 6.5 & 150 & 0 & 5 \\
\hline & & & Placebo & 52 & 1.4 & 4.2 & 76 & 0 & \\
\hline \multirow[t]{2}{*}{ Abou-Alfa et a ${ }^{29}$} & II & 90 & Doxorubicin + sorafenib $400 \mathrm{mg}$ bid po & 66 & 6 & 13.7 & 47 & 3 & 5 \\
\hline & & & Doxorubicin + placebo & 65 & 2.7 & 6.5 & 49 & 2 & \\
\hline \multirow[t]{2}{*}{ Kudo et $\mathrm{a}^{26}$} & III & 458 & Sorafenib $400 \mathrm{mg}$ bid po + TACE & 69 & 5.4 & 29.7 & 229 & 0 & 5 \\
\hline & & & Placebo + TACE & 70 & 3.7 & NR & 229 & 0 & \\
\hline \multirow[t]{2}{*}{ Llovet et $\mathrm{a}^{28}$} & III & 395 & Brivanib $800 \mathrm{mg}$ qd po & 64 & 4.2 & 9.4 & 263 & 6 & 5 \\
\hline & & & Placebo & 62 & 2.7 & 8.2 & 132 & 0 & \\
\hline \multirow[t]{2}{*}{ Kudo et $\mathrm{al}^{27}$} & III & 502 & Brivanib $800 \mathrm{mg}$ qd po & 57 & 12 & 26.4 & 249 & 4 & 5 \\
\hline & & & Placebo & 59 & 10.9 & 26.1 & 253 & 1 & \\
\hline \multirow[t]{2}{*}{ Zhu et $\mathrm{al}^{30}$} & III & 546 & Everolimus 7.5 mg/d & 67 & 3 & 7.6 & 362 & 11 & 5 \\
\hline & & & Placebo & 64 & 2.6 & 7.3 & 184 & 4 & \\
\hline \multirow[t]{2}{*}{ Bruix et al ${ }^{32}$} & III & 1,114 & Sorafenib $400 \mathrm{mg}$ bid po & 58 & 8.5 & NR & 556 & 4 & 5 \\
\hline & & & Placebo & 60 & 8.4 & NR & 558 & 2 & \\
\hline \multirow[t]{2}{*}{ Kang et $\mathrm{al}^{25}$} & ॥ & 202 & Axitinib $5 \mathrm{mg}$ bid po & 61 & 3.6 & 12.7 & 134 & 3 & 5 \\
\hline & & & Placebo & 63 & 1.9 & 9.7 & 68 & 2 & \\
\hline \multirow[t]{2}{*}{ Zhu et $\mathrm{al}^{33}$} & III & 565 & Ramucirumab $8 \mathrm{mg} / \mathrm{kg}$ & 64 & 2.8 & 9.2 & 283 & 7 & 5 \\
\hline & & & Placebo & 62 & 2.1 & 7.6 & 282 & 4 & \\
\hline \multirow[t]{2}{*}{ Bruix et al ${ }^{31}$} & III & 573 & Regorafenib $160 \mathrm{mg}$ & 64 & 3.1 & 10.6 & 379 & 7 & 5 \\
\hline & & & Placebo & 62 & 1.5 & 7.8 & 194 & 2 & \\
\hline
\end{tabular}

Abbreviations: PFS, progression-free survival; OS, overall survival; FAEs, fatal adverse events; qd, once daily; bid, twice daily; po, oral administration; TACE, transarterial chemoembolization; NR, not reported.

and sorafenib showed similar antitumor activity. ${ }^{24}$ Based on these findings, brivanib could not be recommended as first-line treatment for advanced HCC due to its severe toxicities.

\section{Specific cause of FAEs}

Individual specified and non-specified causes of FAEs are listed in Table 2. Of the 45 FAEs on the treatment arms and 17 FAEs on the controlled arms, $13.3 \%$ and $5.9 \%$ were of non-specified etiology, respectively. Of those FAEs that were specified, the most common causes of FAEs associated with MTAs in NSCLC were hepatic failure (22.2\%) and hemorrhage (13.3\%), respectively.

\section{Publication bias}

Begg's funnel plot and Egger's test were performed to assess the publication bias of the literature. There was no evidence of obvious publication bias among included trials for the meta-analysis of FAEs in the present study (Begg's test: $p=0.85$; Egger's test: $p=0.45$, respectively; Figure 4).

\section{Bias analysis}

Randomization procedures and allocation concealment were properly reported in all trials. All of the included trials were double blinded and thus judged to be at low risk of performance and detection bias.

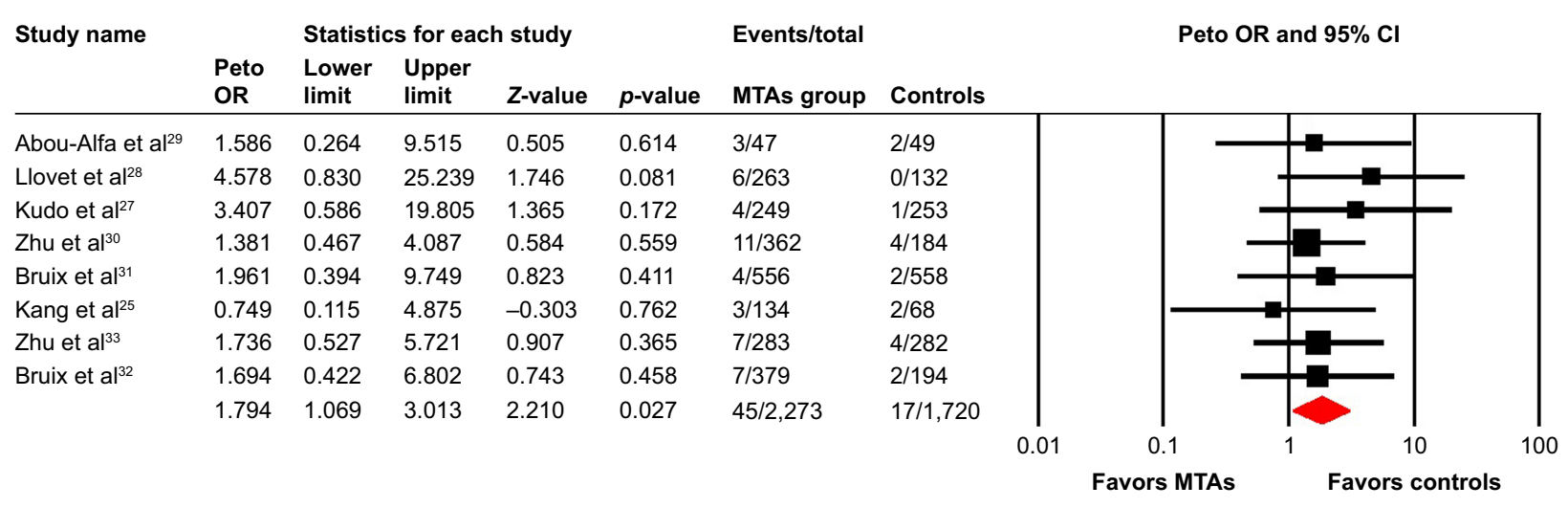

Figure 2 Fixed-effect model of risk ratio $(95 \% \mathrm{Cl})$ of FAEs associated with MTAs in HCC patients.

Abbreviations: FAEs, fatal adverse events; HCC, hepatocellular carcinoma; MTAs, molecular targeted agents. 
Study name

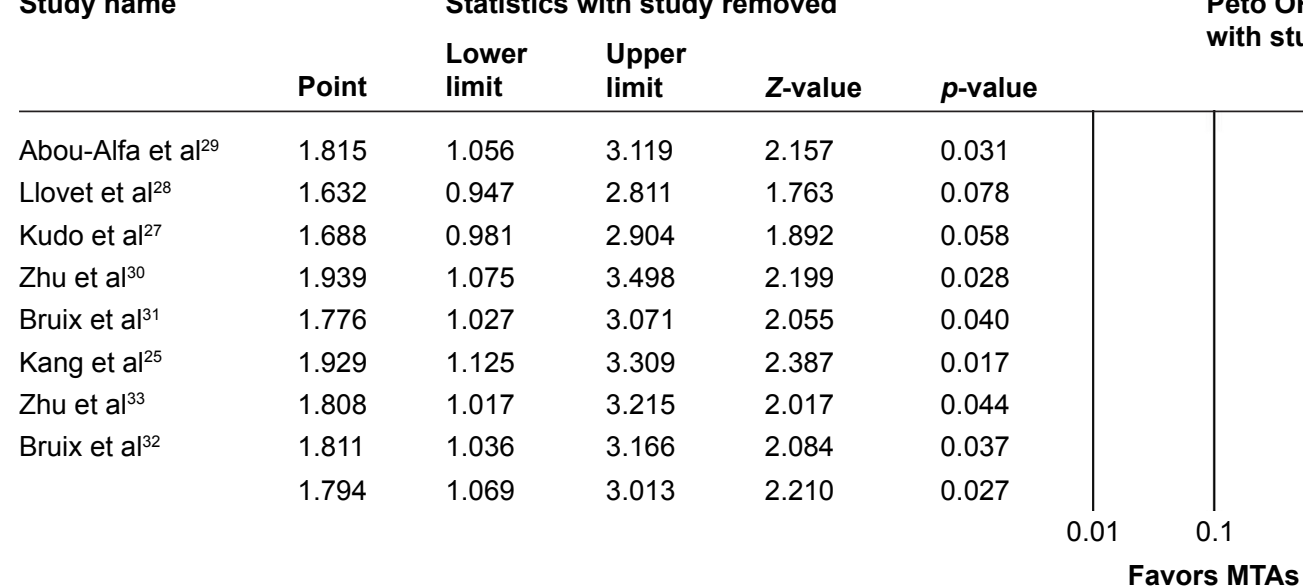

Statistics with study removed

Favors MTAs
Peto OR $(95 \% \mathrm{Cl})$

with study removed

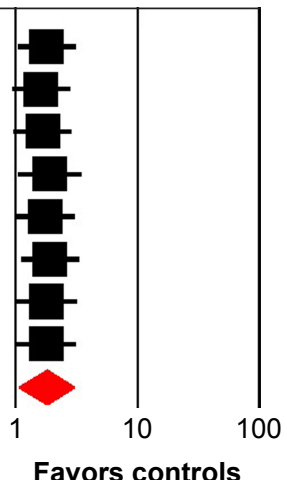

Figure 3 Meta-analysis of FAEs associated with MTAs versus control in HCC patients: "leave-one-out" sensitivity analysis. Abbreviations: FAEs, fatal adverse events; HCC, hepatocellular carcinoma; MTAs, molecular targeted agents.

\section{Discussion}

FAEs are defined as deaths that are secondary to the use of pharmaceutical agents. It is important to monitor the risk of FAEs related to newly targeted agents and to develop risk reduction strategies. Meta-analysis is a powerful statistical tool to estimate the risk of serious drug-related toxicities across a range of medical disciplines by a predetermined method to reduce bias. To our best knowledge, this is the first meta-analysis to evaluate the association between MTAs and FAEs in advanced HCC patients. Based on 10 RCTs involving 4,716 HCC participants, our pooled results demonstrate that the use of MTAs significantly increases the risk of developing FAEs in advanced HCC. However, sensitive

Table 2 Fatal adverse events by specific type

\begin{tabular}{lll}
\hline Adverse events & $\begin{array}{l}\text { Events on the } \\
\text { MTA arms }\end{array}$ & $\begin{array}{l}\text { Events on } \\
\text { control arms }\end{array}$ \\
\hline Death not otherwise specified & $6^{28,30,33}$ & $\mathrm{I}^{32}$ \\
Hepatic failure & $1^{25,27-29,32,33}$ & $5^{25,27}$ \\
Myocardial infarction/ischemia & $3^{29,31}$ & $\mathrm{I}^{32}$ \\
Upper gastrointestinal hemorrhage & $3^{30}$ & $2^{30}$ \\
Intracranial hemorrhage & $3^{32}$ & 0 \\
Encephalopathy & $3^{28}$ & 0 \\
Renal failure & $3^{30,33}$ & $\mathrm{I}^{30}$ \\
Pulmonary infection, pneumonitis & $3^{25,27,30}$ & $\mathrm{I}^{25}$ \\
Gastric perforation & $\mathrm{I}^{30}$ & 0 \\
Febrile neutropenia & 0 & $\mathrm{I}^{29}$ \\
Thrombosis or embolism & 0 & $2^{29,33}$ \\
Acidosis & $\mathrm{I}^{28}$ & 0 \\
Cerebral edema & $\mathrm{I}^{28}$ & 0 \\
Cerebrovascular accident & $\mathrm{I}^{30}$ & 0 \\
Interstitial lung disease & $\mathrm{I}^{30}$ & 0 \\
Peritonitis & $2^{27,30}$ & 0 \\
Respiratory failure & $\mathrm{I}^{30}$ & $\mathrm{I}^{30}$ \\
Multiorgan failure & $\mathrm{I}^{33}$ & $\mathrm{I}^{30}$ \\
Sepsis, septic shock & $2^{25,33}$ & $\mathrm{I}^{33}$ \\
\hline Abrevation: MTA, mo &
\end{tabular}

Abbreviation: MTA, molecular targeted agent. analysis shows that the pooled results are influenced by removing a single trial, which suggest that further studies are still needed to clearly determine the risk of FAEs in HCC patients. The summary incidence of FAEs is $2.1 \%$. It should be mentioned that all FAE data in the present study are derived from clinical trials with rigorous inclusion criteria. Therefore, the risk of FAEs may be even higher in conventional medical practice.

As HCC patients receiving different MTAs are included, this might influence the incidence and risk of FAE analysis. Subgroup analysis according to the specific targeted drugs shows that the use of brivanib in HCC significantly increases the risk of FAEs (Peto OR 3.97, $p=0.028$ ). Therefore, clinicians should pay more attention to drug-related toxicities, but not only for antitumor activity. Indeed, toxicity is a significant concern during the development of novel targeted agents for advanced HCC. A Phase III RCT in the first-line setting with a head-to-head comparison between the sunitinib and sorafenib was prematurely discontinued following a review by the Independent Data Monitoring Committee (IDMC) owing to safety concerns (more frequent and severe AEs) in the sunitinib arm and the lack of either superiority or non-inferiority in OS compared with sorafenib ( 7.9 vs 10.2 months, two-sided $p=0.0014) .{ }^{23}$ In another Phase III head-to-head trial comparing linifanib versus sorafenib as first-line treatment for advanced HCC, the authors also found that linifanib was more toxic than sorafenib in terms of serious AEs ( $54 \%$ vs $38 \%$ ), grade 3 to 4 hypertension ( $20 \%$ vs $10 \%$ ) and grade 3 to 4 liver-related complications (encephalopathy, ascites, and hyperbilirubinemia: $20 \%$ vs $10 \%) .{ }^{22}$ Although the reasons for these two Phase III trials failure are heterogeneous, the major treatment failure reasons include drug-related toxicities 


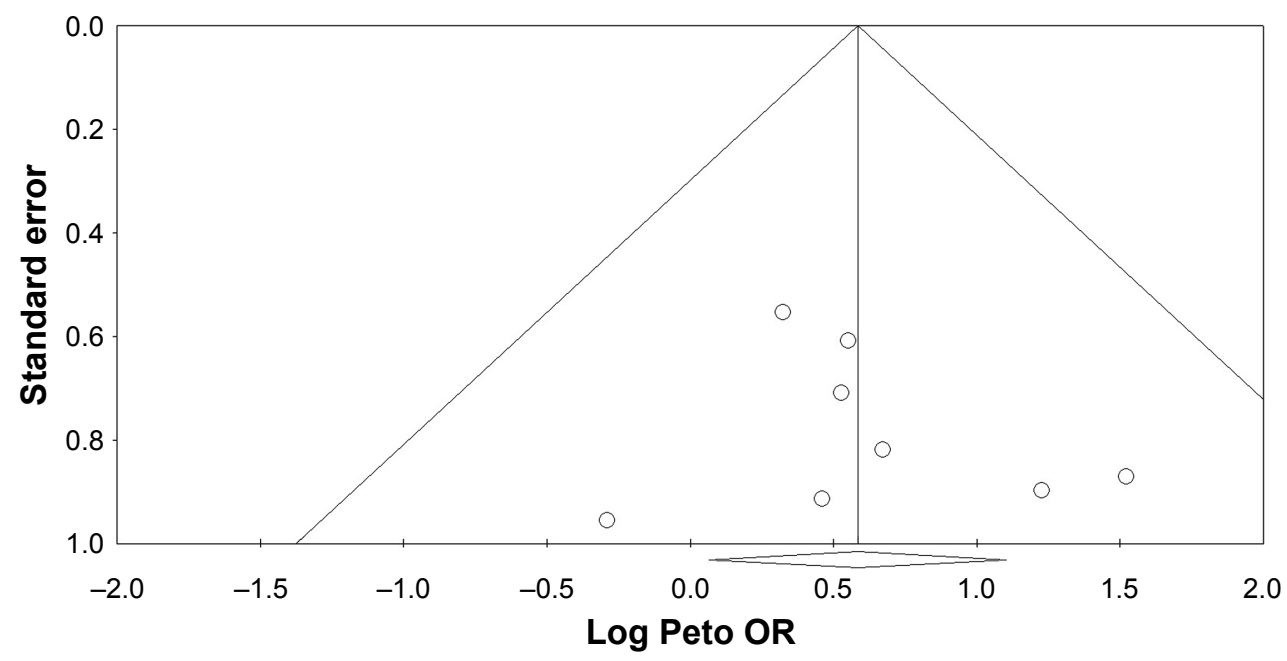

Figure 4 Funnel plot for publication bias.

and lack of substantial antitumoral potency. Preclinical studies found that upregulation of pro-angiogenic signals such as the fibroblast growth factor receptor (FGFR) signaling pathway might have a crucial role in the acquired resistance to sorafenib; thus, the dual VEGFR and FGFR TKI brivanib was one of the most tested in Phase III RCTs for HCC. However, the first-line trial of a head-to-head comparison with sorafenib did not meet its primary end point of demonstrating non-inferiority in OS, despite similar results in secondary end points (time to progression, objective response rate, disease control rate assessed by modified RECIST). ${ }^{24}$ In addition, brivanib was not as well tolerated as sorafenib, with a higher rate of serious AEs and AEs leading to discontinuation. Based on these findings, it seems likely that the marginal differences in OS between the various molecular drugs that are failed in Phase III trials might be attributable to differences in toxicity. As a result, delineated strategies to guide development of other novel drugs, including better monitoring of treatment-related AEs, and optimized dose selection for the cirrhotic population, might be a turning point in the clinical management of advanced HCC.

Of the reported specified causes of FAEs, the incidence of hepatic failure (22.2\%) and hemorrhage (13.3\%) are numerically higher in the MTAs group. This result is consistent with a previous meta-analysis, which concluded that targeted agents in $\mathrm{HCC}$ were associated with increased risk of all grade and high grade hemorrhage. ${ }^{34}$ If any bleeding necessitates medical intervention, permanent discontinuation of sorafenib should be considered according to the manufacturer's instructions. TKI-induced hepatitis is characterized by a hepatocellular pattern of liver damage with significant increases of transaminases which may result in hepatic failure and death. It has been recommended to regularly monitor liver function test for patients receiving TKIs. In the case of significantly increased transaminases without alternative explanation, such as viral hepatitis or progressing underlying malignancy, TKIs should be discontinued according to the manufacturer's instructions.

Our study has several limitations. First, FAE data were abstracted from clinical trial results; therefore, we could not get individual patient information. Second, FAEs are not the primary end point in all included RCTs, and the process of attribution of FAEs' causality by investigators is an objective process and may be a potential source of bias. However, the majority of included RCTs in our study are double blinded and placebo controlled and thus are determined to be of low risk of bias. Furthermore, in our sensitivity analysis that excluded open-label trials and unpublished RCTs, we did not observe any significant change in the pooled ORs.

\section{Conclusion}

The present study has found that the use of MTAs significantly increases the risk of developing FAEs. Given the increasing use of MTAs in HCC patients, clinicians should be aware of the risks of FAEs with MTAs treatment in HCC patients and prevent accordingly, especially those caused by liver toxicity.

\section{Disclosure}

The authors report no conflicts of interest in this work.

\section{References}

1. Jemal A, Bray F, Center MM, Ferlay J, Ward E, Forman D. Global cancer statistics. CA Cancer J Clin. 2011;61(2):69-90. 
2. Siegel R, Naishadham D, Jemal A. Cancer statistics, 2013. CA Cancer J Clin. 2013;63(1):11-30.

3. Giannini EG, Farinati F, Ciccarese F, et al. Prognosis of untreated hepatocellular carcinoma. Hepatology. 2015;61(1):184-190.

4. Han P, Li H, Jiang X, et al. Dual inhibition of Akt and c-Met as a secondline therapy following acquired resistance to sorafenib in hepatocellular carcinoma cells. Mol Oncol. 2017;11(3):320-334.

5. Rota Caremoli E, Labianca R. Tivantinib: critical review with a focus on hepatocellular carcinoma. Expert Opin Investig Drugs. 2014;23(11): $1563-1574$.

6. Zhai B, Sun XY. Mechanisms of resistance to sorafenib and the corresponding strategies in hepatocellular carcinoma. World $J$ Hepatol. 2013;5(7):345-352.

7. Torrecilla S, Llovet JM. New molecular therapies for hepatocellular carcinoma. Clin Res Hepatol Gastroenterol. 2015;39 Suppl 1: S80-S85.

8. Cheng AL, Kang YK, Chen Z, et al. Efficacy and safety of sorafenib in patients in the Asia-Pacific region with advanced hepatocellular carcinoma: a phase III randomised, double-blind, placebo-controlled trial. Lancet Oncol. 2009;10(1):25-34.

9. Llovet JM, Ricci S, Mazzaferro V, et al. Sorafenib in advanced hepatocellular carcinoma. N Engl J Med. 2008;359(4):378-390.

10. Zhang XJ, Zhang TY, Yu FF, et al. Risk of treatment-related mortality with sorafenib in patients with cancer. Asian Pac J Cancer Prev. 2014; 14(11):6681-6686.

11. Sonpavde G, Je Y, Schutz F, et al. Venous thromboembolic events with vascular endothelial growth factor receptor tyrosine kinase inhibitors: a systematic review and meta-analysis of randomized clinical trials Crit Rev Oncol Hematol. 2013;87(1):80-89.

12. Qi WX, Min DL, Shen Z, et al. Risk of venous thromboembolic events associated with VEGFR-TKIs: a systematic review and meta-analysis. Int J Cancer. 2013;132(12):2967-2974.

13. Wesolowski R, Abdel-Rasoul M, Lustberg M, Paskell M, Shapiro CL, Macrae ER. Treatment-related mortality with everolimus in cancer patients. Oncologist. 2014;19(6):661-668.

14. Gu B, Gao W, Chu H, et al. Adverse events risk associated with antiVEGFR agents in the treatment of advanced nonsmall-cell lung cancer: a meta-analysis. Medicine (Baltimore). 2016;95(48):e3752.

15. Zhang D, Ye J, Xu T, Xiong B. Treatment related severe and fatal adverse events with cetuximab in colorectal cancer patients: a metaanalysis. J Chemother. 2013;25(3):170-175.

16. Choueiri TK, Je Y, Sonpavde G, et al. Incidence and risk of treatmentrelated mortality in cancer patients treated with the mammalian target of rapamycin inhibitors. Ann Oncol. 2013;24(8):2092-2097.

17. Sivendran S, Liu Z, Portas LJ Jr, et al. Treatment-related mortality with vascular endothelial growth factor receptor tyrosine kinase inhibitor therapy in patients with advanced solid tumors: a meta-analysis. Cancer Treat Rev. 2012;38(7):919-925.

18. Moher D, Cook DJ, Eastwood S, Olkin I, Rennie D, Stroup DF. Improving the quality of reports of meta-analyses of randomised controlled trials: the QUOROM statement. Quality of reporting of meta-analyses. Lancet. 1999;354(9193):1896-1900.

19. Sweeting MJ, Sutton AJ, Lambert PC. What to add to nothing? Use and avoidance of continuity corrections in meta-analysis of sparse data. Stat Med. 2004;23(9):1351-1375.
20. Zintzaras E, Ioannidis JP. Heterogeneity testing in meta-analysis of genome searches. Genet Epidemiol. 2005;28(2):123-137.

21. Moher D, Pham B, Jones A, et al. Does quality of reports of randomised trials affect estimates of intervention efficacy reported in meta-analyses? Lancet. 1998;352(9128):609-613.

22. Cainap C, Qin S, Huang WT, et al. Linifanib versus sorafenib in patients with advanced hepatocellular carcinoma: results of a randomized phase III trial. J Clin Oncol. 2015;33(2):172-179.

23. Cheng AL, Kang YK, Lin DY, et al. Sunitinib versus sorafenib in advanced hepatocellular cancer: results of a randomized phase III trial. J Clin Oncol. 2013;31(32):4067-4075.

24. Johnson PJ, Qin S, Park JW, et al. Brivanib versus sorafenib as firstline therapy in patients with unresectable, advanced hepatocellular carcinoma: results from the randomized phase III BRISK-FL study. J Clin Oncol. 2013;31(28):3517-3524.

25. Kang YK, Yau T, Park JW, et al. Randomized phase II study of axitinib versus placebo plus best supportive care in second-line treatment of advanced hepatocellular carcinoma. Ann Oncol. 2015;26(12): 2457-2463.

26. Kudo M, Imanaka K, Chida N, et al. Phase III study of sorafenib after transarterial chemoembolisation in Japanese and Korean patients with unresectable hepatocellular carcinoma. Eur J Cancer. 2011;47(14): 2117-2127.

27. Kudo M, Han G, Finn RS, et al. Brivanib as adjuvant therapy to transarterial chemoembolization in patients with hepatocellular carcinoma: a randomized phase III trial. Hepatology. 2014;60(5):1697-1707.

28. Llovet JM, Decaens T, Raoul JL, et al. Brivanib in patients with advanced hepatocellular carcinoma who were intolerant to sorafenib or for whom sorafenib failed: results from the randomized phase III BRISK-PS study. J Clin Oncol. 2013;31(28):3509-3516.

29. Abou-Alfa GK, Johnson P, Knox JJ, et al. Doxorubicin plus sorafenib vs doxorubicin alone in patients with advanced hepatocellular carcinoma: a randomized trial. JAMA. 2010;304(19):2154-2160.

30. Zhu AX, Kudo M, Assenat E, et al. Effect of everolimus on survival in advanced hepatocellular carcinoma after failure of sorafenib: the EVOLVE-1 randomized clinical trial. JAMA. 2014;312(1):57-67.

31. Bruix J, Qin S, Merle P, et al. Regorafenib for patients with hepatocellular carcinoma who progressed on sorafenib treatment (RESORCE): a randomised, double-blind, placebo-controlled, phase 3 trial. Lancet. 2017;389(10064):56-66.

32. Bruix J, Takayama T, Mazzaferro V, et al. Adjuvant sorafenib for hepatocellular carcinoma after resection or ablation (STORM): a phase 3, randomised, double-blind, placebo-controlled trial. Lancet Oncol. 2015;16(13):1344-1354.

33. Zhu AX, Park JO, Ryoo BY, et al. Ramucirumab versus placebo as second-line treatment in patients with advanced hepatocellular carcinoma following first-line therapy with sorafenib (REACH): a randomised, double-blind, multicentre, phase 3 trial. Lancet Oncol. 2015;16(7):859-870.

34. Duffy A, Wilkerson J, Greten TF. Hemorrhagic events in hepatocellular carcinoma patients treated with antiangiogenic therapies. Hepatology. 2013;57(3):1068-1077.

\section{Publish your work in this journal}

Drug Design, Development and Therapy is an international, peerreviewed open-access journal that spans the spectrum of drug design and development through to clinical applications. Clinical outcomes, patient safety, and programs for the development and effective, safe, and sustained use of medicines are the features of the journal, which

\section{Dovepress}

has also been accepted for indexing on PubMed Central. The manuscript management system is completely online and includes a very quick and fair peer-review system, which is all easy to use. Visit http://www.dovepress.com/testimonials.php to read real quotes from published authors. 